\title{
Why is Mars the "Red Planet"? A New, Novel Hypothesis on the Features of Mars and the Origin of the Asteroid Belt
}

\author{
Jack R. Edelman ${ }^{1}$ \\ ${ }^{1}$ Department of Science, Borough of Manhattan Community College, The City University of New York, 199 \\ Chambers Street, New York, New York, 10007, U.S.A. \\ Correspondence: Jack R. Edelman, Department of Earth \& Environmental Sciences (EES), Brooklyn College, \\ The City University of New York, 2900 Bedford Avenue, Brooklyn, New York, 11210, U.S.A., (candidate for \\ Master of Arts degree in EES). E-mail: RJNobleman@aol.com
}

Received: December 7, 2021

Accepted: January 28, 2022

Online Published: February 14, 2022

doi: $10.5539 /$ jgg.v14n1p1

URL: https://doi.org/10.5539/jgg.v14n1p1

\begin{abstract}
Mars has always been known as the "Red Planet" because it is the only planet in our solar system with a red surface. The reasons for this uniqueness have never been proposed, other than the fact that its soil and surface rocks have a high content of iron oxide. In order to attempt to resolve this issue, a novel hypothesis is proposed herewith. Formulation of this hypothesis involved considering the unique features of Mars and putting them into a rational correlating explanation. The scenario involves the collision of a formerly much larger Mars with another planet early in the solar system's history. The crust and mantle were blown off, forming the asteroid belt, leaving the iron core intact. Upon cooling and solidification, the iron core combined with oxygen in the remaining atmosphere to form rust-colored iron oxide (rust). Methane in the early atmosphere underwent combustion, forming its current high carbon dioxide atmosphere. The combustion of methane also produced massive amounts of liquid water, explaining the dried-up erosional features of the planet's surface, such as river valleys. The point of impact formed a long, deep canyon, Valles Marineris, and the nearby giant volcano, Olympus Mons, even in the absence of Mars' tectonic activity. The cooled, solidified core may explain why Mars has no magnetic field. If accurate, these ideas may help space programs prepare astronauts for manned exploration of the Red Planet, as well as facilitating an understanding of its unusual features.
\end{abstract}

Keywords: Mars, Red Planet, Olympus Mons, Valles Marineris, Asteroid belt, Magnetic Field, Tharsis Region, Tectonics

\section{Introduction/Background}

Mars has always been known as "The Red Planet" due to its red color when seen through the telescope and/or the naked eye. The reasons for its red color have been attributed to the high iron oxide content of its soil and surface rocks. Few scientists have attempted to explain, however, why it seems to be the only planet in the solar system with a red surface while the other eight (or seven if one doesn't consider Pluto a regular planet) do not possess this red coloration nor why the soil and surface rocks do not have such have a high content of iron oxide compared to Mars. Some hypotheses suggest that this is due to the relatively small size of Mars, which, due to resultant lower gravity, failed to pull iron toward its core during the accretion process (Wolchover, 2012) This idea, however, should also cause Mercury to be a 'red planet', yet it is not. This report describes a new, novel and unusual hypothesis to resolve this enigma of why Mars is the 'red planet', as well as the origin of the asteroid belt.

\section{Formulation of the Hypothesis}

The planet Mars is unique in a number of characteristics, as follows:

(1) Its red soil and surface rocks, due to a high content of iron oxide

(2) The surface rocks are angular in shape and of different sizes

(3) The atmosphere is mainly carbon dioxide

(4) It appears to have had much flowing surface water in the distant past

(5) It has little or no magnetic field/magnetosphere 
(6) It lies near the asteroid belt between itself and Jupiter

(7) It exhibits dust storms on its surface

(8) It seems to have no tectonic activity

(9) It has the solar system's largest volcano (now extinct), Olympus Mons

(10) The few volcanoes (all currently extinct) that it does have are mainly or exclusively confined to a special area, the Tharsis region

(11) It contains a large, possibly the deepest canyon in the solar system, Valles Marineris

(12) It has two moons, Phobos and Deimos, which are unusual in size (small) and shape; these are most likely captured asteroids

(13) Mars is a member of the inner, rocky planets (Mercury-Venus-Earth-Mars, in that order of distance from the sun). The sizes (diameters) of the inner, rocky planets (Mercury-Venus-Earth) increase in size progressively in that same order, yet Mars breaks this progression by being smaller than Earth.

The novel hypothesis to be described conforms with the unique features of Mars described above.

\section{The Proposed Hypothesis}

According to this new idea, originally proposed in a much simpler form several years ago (Edelman, 2012), Mars was originally a much larger planet. There was some kind of 'cosmic collision' earlier in the history of the solar system, possibly with another planet or comet, or other heavenly body. During that purported collision, the crust and mantle of Mars were blown away, leaving only the hot iron/nickel core intact, with possibly some remnants of the crust and/or mantle remaining, along with the original atmosphere, which was largely methane (like the outer gas giants, Jupiter, Uranus, Neptune). Over time, the surface of the iron/nickel core reacted with the remaining oxygen in the atmosphere, forming iron oxide (red and yellow rusts). The remining methane also underwent combustion with the oxygen, forming carbon dioxide and water. The blown-off crust and mantle were flung into space, forming the asteroid belt. Two asteroids became the moons, Phobos and Deimos.

\section{Discussion}

Now we must consider and elaborate on each of the above unusual characteristics of the planet used to formulate the novel hypothesis:

(1) The red soil and rocks are believed to owe their color to a high composition of iron oxide (rust) (Wolchover, 2012; Kaufman, 2014). This might be explained, correlating with the hypothesis, by oxidation of the iron/nickel core surface (rusting) over time. If the original atmosphere contained appreciable amounts of oxygen, and if some oxygen remained after the blast (collision), there could have been oxidation of the iron, forming the red and possibly yellow color of the soil and surface rocks we see today. Water that might have been formed by combustion of methane during the collision could have dissolved some of the atmospheric oxygen, further resulting in oxidation/rusting of the core surface.

(2) The angular, irregular shapes and varying sizes of the surface rocks can be explained by the blast/collision. These features of the surface rocks can easily be noted in photos supplied by Darling (2022). In this photo, all rocks are angular in shape. Smooth, rounded rocks that would be formed from erosion by the flow of running water are not seen in this photo. This lends supporting evidence to the idea that the surface rocks were formed during some type of blast or other violent cataclysm. 


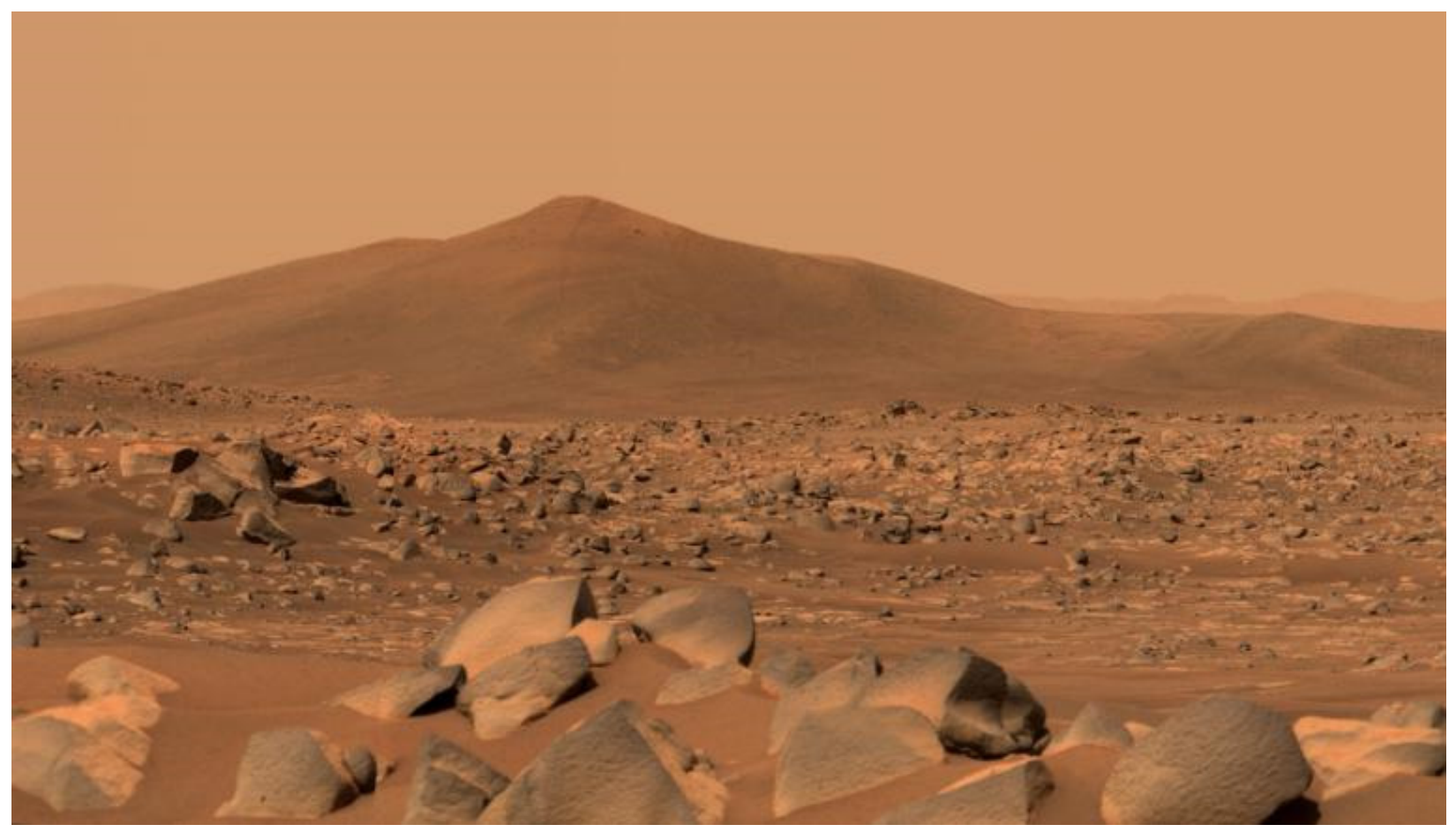

Figure 1. Rocks on the surface of Mars. Note various sizes and shapes (boulders and cobbles), all of which contain sharp, angular edges, characteristic of a tremendous blast. Photo courtesy of NASA. Reprinted with permission.

(3) The fact that Mars has an atmosphere high in carbon dioxide (Sharp, 2017) might be explained by this hypothesis if the original atmosphere was high in methane content, like its nearby gas giants (Jupiter, Saturn, Uranus, Neptune). If large amounts of oxygen were also present, the collision could have generated enough heat/energy to cause the methane/other hydrocarbons to combust, forming carbon dioxide and water.

(4) The flowing water that scientists believe used to exist on Mars (Goudge, et al., 2018) but are no longer present on its surface in any appreciable amounts, could be explained by rationale number 3 above. If methane/other hydrocarbons were present and combustion took place by the collision, much water would have been produced in addition to carbon dioxide. And if large volumes of methane/other hydrocarbons were indeed present as well as large volumes of oxygen, large volumes of water could account for the lakes, rivers, and streams (surface waters) that purportedly existed there, until they evaporated and were lost to space or percolated through the soil into groundwater.

(5) Mars' lack or reduction of a magnetic field/magnetosphere (Zhong, 2020) might be explained by this hypothesis by cooling and solidification of the iron/nickel core over time. In order for a planet to have a magnetosphere, it must have an iron/nickel core which is liquid and dynamic/moving (Ouladdiaf, 2022). If the iron/nickel core cooled and solidified, as this hypothesis assumes, that would account for the absence of a magnetosphere on present day Mars.

(6) and (12) The asteroid belt lies only between Mars and Jupiter and is found between no other planets (although an occasional escaped asteroid is seen in other areas of the solar system). The explanation for this hitherto originally was that there had originally been another planet in that region that somehow exploded (Learn, 2021). This idea has been changed to the idea that there originally had been planetesimals in that region but, due to the pull of Jupiter's gravity and the low gravity of Mars, were not able to accrete into an actual planet (Choi, 2021). According to the hypothesis proposed herewith, the asteroid belt might have been formed by the purported collision, which blasted the crust and mantle of ancient Mars into space. Some of the blasted rock may have fell onto the surface, which could explain the sharp, irregular rocks described in item number 1 above, and visible from the photo provided by Darling (2022). In addition, Mars' two moons, Phobos and Deimos, might be explained as asteroids/ material that re-entered its orbit and were permanently re-captured (Choi, 2021).

(6) Surface dust storms might be explained by a similar occurrence on Earth. In the Midwest region of the United States during the 1940s (Trimarchi, 2022). During this period, fine soil and loess particles caused by topsoil erosion and weathering due to over-farming and non-rotation of crops were blown for long distances by 
wind. Their pulverized, miniscule dust-like size and weight allowed winds to blow them into tremendous storms of 'dust'. If, according to the proposed new hypothesis, the solidified iron core oxidized with the remaining atmospheric oxygen into iron oxide rust, which is powdery and light in weight, it could easily be blown long distances by wind, forming the patches we see today and which were originally proposed to be 'canals' (Vivid Maps, 2022).

(8-11). There is little, if any, tectonic activity on Mars, probably none at all (Wikipedia, 2022). As a result, the question arises as to how the few (now extinct) volcanoes it does have were produced and why Olympus Mons seems to be the largest volcano in the solar system. Looking at maps of Mars, we can see that these volcanoes, as well as Olympus Mons, is relatively nearby to Valles Marineris. This is also shown in photos and drawings and other images provided by Coles, et al., (2019). This might be explained by this hypothesis, if Valles Marineris represents the point of impact of the colliding planet. The heat/energy produced by the purported impact could have melted the region directly below, and this might account for the vulcanism and for the huge size of Olympus Mons, especially in the absence of tectonic activity, and the relative proximity of this chasm to this (Tharsis) volcanic region (Stack Exchange,2022). The actual origin of Valles Marineris remains uncertain, as noted by Coles et al., (2019). The hypothesis proposed herewith, i.e., that it was formed by a cosmic collision, offers a possible explanation.

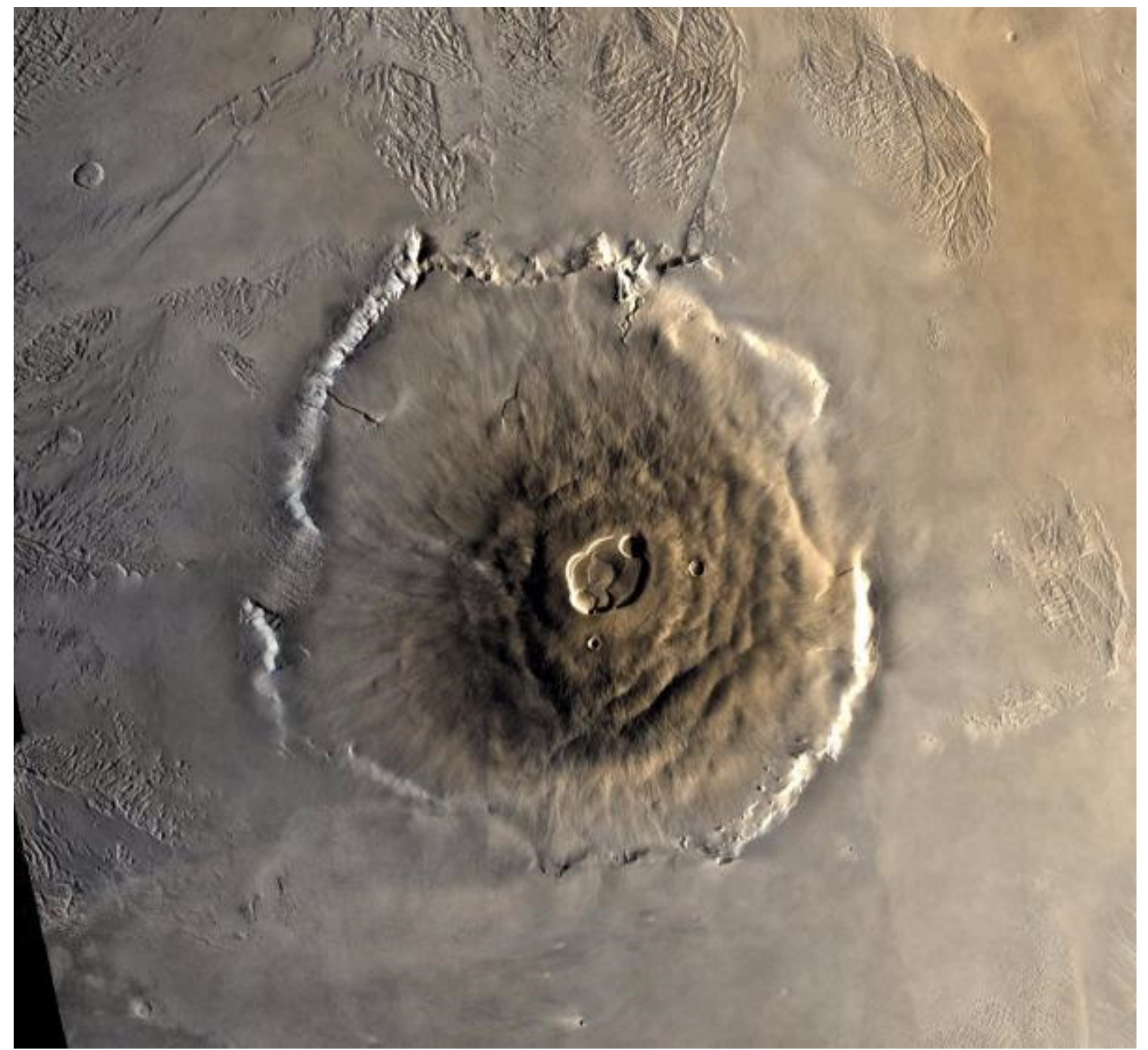

Figure 2. Olympus Mons, the largest volcano in our solar system, formed despite the lack of tectonic activity. Located in relatively close proximity to Valles Marineris, might it be due to heating and melting of the surface and/or sub-surface due to the purported collision? Photo courtesy of NASA. Reprinted with permission. 


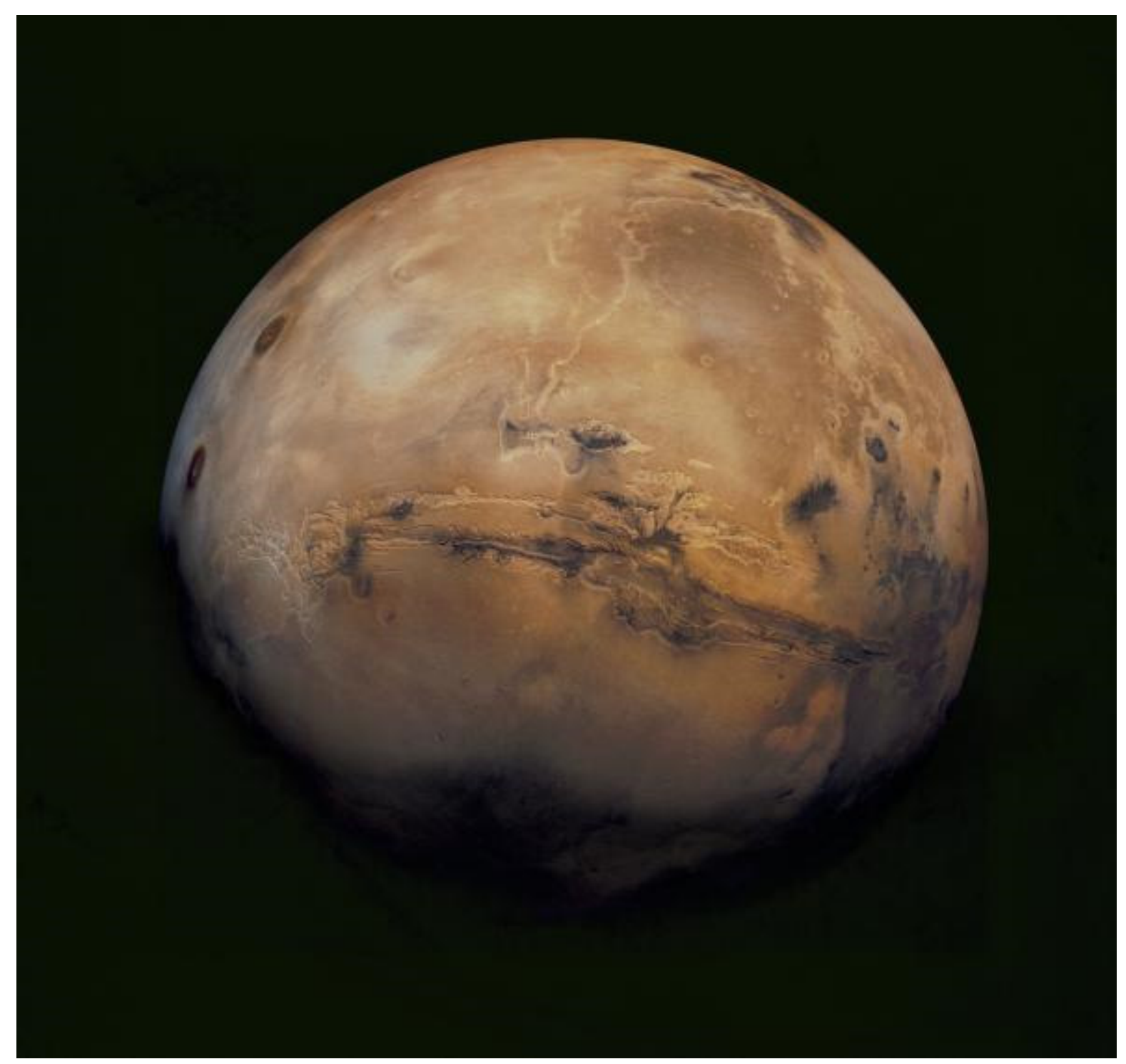

Figure 3. Valles Marineris. A huge chasm on Mars' surface. Might it be the site of an ancient collision? Large circular depression in the center may be the actual point of the purported collision. Large circular depression in center appears to exist nearly midway between right and left boundaries of the chasm, which would be expected by an impact as the origin of the chasm. Photo courtesy of NASA, reprinted with permission. 


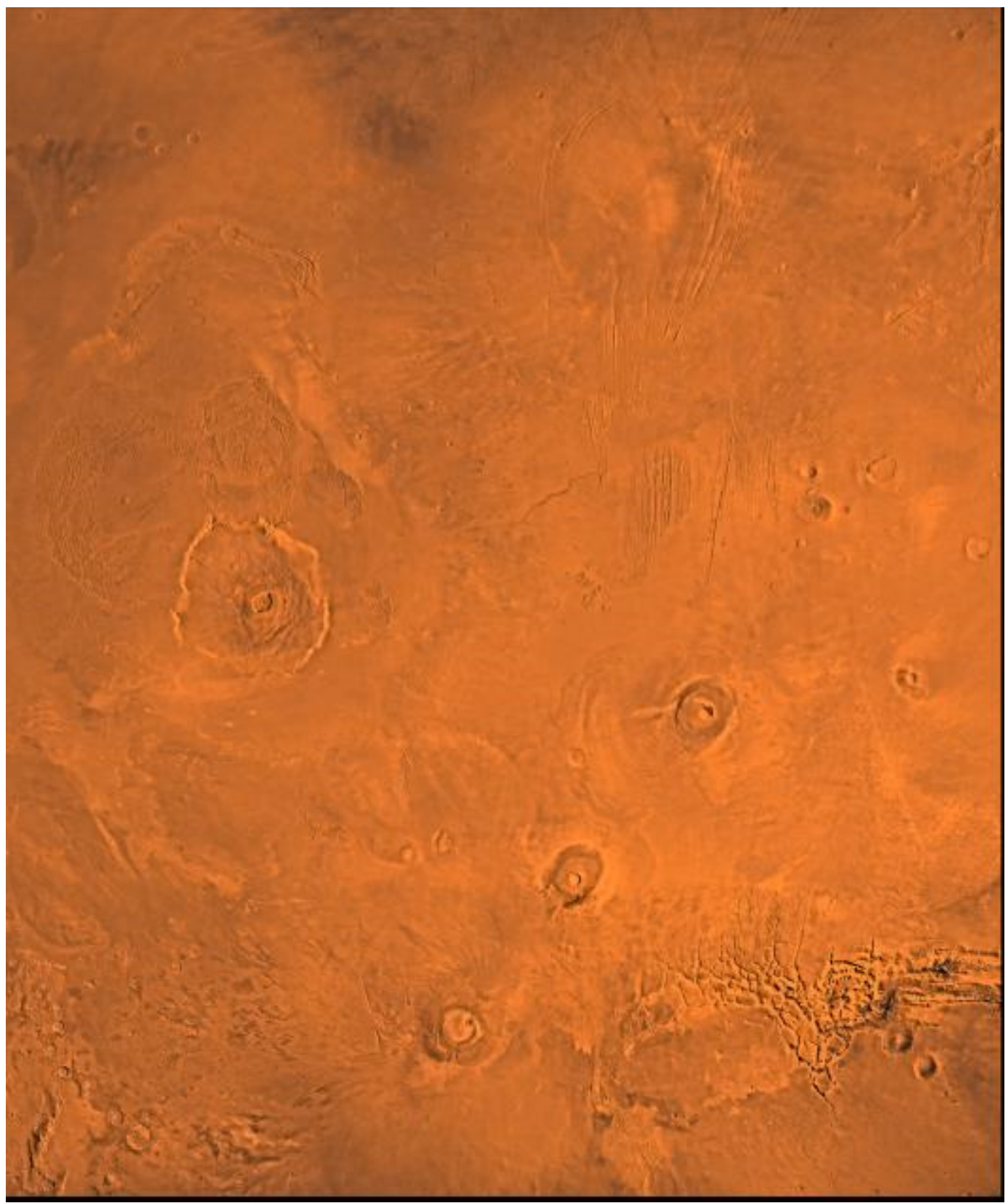

Figure 4. Most Martian volcanoes, including Olympus Mons, are located in the Tharsis region, relatively close to Valles Marineris, the purported site of collision according to this novel hypothesis. Note diagonal chain of volcanoes near center. Note also beginning of Valles Marineris chasm at lower right, in relatively close proximity to the volcanic chain. Heat generated by the nearby collision may account for subsurface melting and production of the volcanic chain in the absence of tectonic activity. Photo courtesy of NASA. Reprinted with permission.

(13) Mars is the last in the series of the inner, rocky planets. The order of decreasing distance from the Sun is Mercury $<$ Venus $<$ Earth $<$ Mars. Interestingly, with increasing distance from the Sun, the size (diameter of the planets) usually increases (Earth $>$ Venus $>$ Mercury). If this progressive increase in diameter is the 'norm', then, theoretically, at least, Mars should have the largest size (diameter) of the four inner planets, since it has the largest distance from the Sun. Yet, Mars does NOT have the largest diameter; it is smaller than the Earth (Williams, 2022). This might imply that Mars originally was a larger planet than Earth, according to the hypothesis. This idea might lend supporting evidence to the hypothesis that Mars' size was reduced, most likely 
after some type of cosmic collision. This idea of increasing planetary diameter with distance from the Sun, on the other hand, does not seem to correlate for the outer gas giants, since Jupiter then would be larger than Neptune (the idea of Pluto being a 'planet' in this case would be excluded). However, the gas giant planets are substantially larger than the Earth and all the inner, rocky planets, indicating that mars should, have according to the hypothesis, been much larger than it currently is. It is also of interest, possibly related to this hypothesis, that a major theory regarding the origin of the Earth's moon is that a Mars-sized body collided with the earth early in its history. The collision may have ejected material into Earth's orbit, forming the moon (Lotzoff, 2022).

\section{Conclusions}

This new and unusual hypothesis attempts to explain the features and properties of the planet Mars (the "Red Planet"), including the reason it is red and the only planet in our solar system that is indeed that color. The assumptions/speculations of this hypothesis are as follows:

(1) The Mars we see today is not the same planet in its original form. It is the remains of a much larger planet in which the crust and mantle were blown off in some type of prehistoric collision/cataclysm with another planet or large heavenly body. (2) The blown-off crust and mantle were thrust into space and formed the asteroid belt. (3) The iron/nickel core cooled and solidified, with the surface oxidizing with remaining atmospheric oxygen to become iron oxide (rust). This is what gives the surface its red or red/brown color. (4) The collision produced a huge surface chasm at the point of impact which we know as Valles Marineris. The heat/energy produced from the impact melted the region below, forming the Tharsis region, with several volcanoes, including the largest volcano in the solar system, Olympus Mons. All such volcanoes are now extinct. This would explain the collection of volcanoes in the Tharsis region in the absence of plate tectonics, as well as the relative proximity of these volcanoes to Valles Marineris. (5) The high methane and oxygen of the primitive atmosphere reacted during this collision by combustion, forming the high carbon dioxide atmosphere and large amounts of flowing surface water, which has since dried up and/or percolated below the surface. (6) oxygen remaining in the atmosphere oxidized the iron on the surface of the remaining core, forming the iron-oxide-rich red soil and rock. (7) As the core lost heat to space, it solidified, causing loss of the planets magnetosphere. If this hypothesis is valid/accurate, it might help our future space programs, insofar as research on Mars is concerned as well as future planned manned missions to the Red Planet. It may also help scientists understand the enigmatic properties of the planet. Since Mars is nick-named for the "God of War", and according to this hypothesis may be the rusty core of an pre-existing, larger planet, another nickname hereby proposed for the "Red Planet" might be "The Rusty warrior"!

\section{Conflict of interest}

The author declares that there is no conflict of interests regarding the publication of this paper.

\section{Acknowledgements}

Gratitude is expressed to Ms. Connie Moore, National Aeronautics and Space Administration (NASA) for providing and granting permission to use the images shown in the figures of this paper.

\section{References}

Choi, C.Q. (2021). Were the Mars moons Phobos and Deimos born from another Shattered Moon? https://www.space.com/mars-moons-phobos-deimos-shattered-moon-origins

Coles, K.S., Tanaka, K.L., Christensen, P.R. (2019). The Atlas of Mars. Mapping Its Geography and Geology. Cambridge University Press, Cambridge, England. ISBN 978-1-107-03629-1.

Daniels, P. (2010). The New Solar System. Ice Worlds, Moons, and Planets Redefined. Expanded Edition. National Geographic Society, Washington, D.C. ISBN 978-1-4262-0752-5.

Darling, D. (2022). Retrieved from: www.daviddarling.info/images/Mars_panorama.jpg

Edelman, J/R. (2012). Mars is NOT the Red Planet. Retrieved from http://www.crazyhypotheses.net

Goudge, T.A., Mohrig, D., Cardenas, B.T., Hughes, C.M., Fassett, C.I. (2018). Stratigraphy and Paleohydrology of Delta Channel Deposits, Jezero Crater, Mars. Icarus, 301: 58-75. https://doi.org/10.1016/j.icarus.2017.09.034

Kaufman, M. (2014) MARS UP CLOSE INSIDE THE CURIOSITY MISSION. National Geographic Society, Washington, D.C. ISBN 978-1-4262-1278-9.

King, I.R. (1976). The Universe Unfolding. W.H. Freeman and Co. San Francisco, CA ISBN 0-7167-0521-4 Learn, J.R., (2021). The Asteroid Belt: Wreckage of a Destroyed Planet or something Else? Retrieved from 
https://www.discovermagazine.com/the-sciences/the-asteroid-belt-wreckage-of-a-destroyed-planet-or-some thing-else

Lotzoff, R., (2022). How Did the Moon Form? Retrieved from: https://www.nhm.ac.uk/discover/how-did-the-moon-form.html

Ouladdiaf, B. (2022). How Mars lost its magnetism. Retrieved from: https:/www.ill.eu/fileadmin/user_upload/ILL/3_Users/Scientific_groups/Diffraction/Home/mars.pdf

Sharp, T. (2017) Mars' Atmosphere: Composition, Climate, \& weather. Retrieved From https:/www.space.com/16903-mars-atmosphere-climate-weather.html

Sparrow, G. (2015). MARS. A New View Of The Red planet. Quercus Publishing, New York. ISBN 978-1-62365-856-4.

StackExchange. (2022). Space Exploration Beta. Retrieved $\mathrm{https}$ ://space.stackexchange.com/how-to-determine-the-distance-between-olympus-mons-and-valles-marine ris

Trimarchi, M. (2021). Midwest Dust Bowl: What Caused the Dust Bowl? Retrieved From: https://science.howstuffworks.com/environmental/green-science/dust-bowl-cause.html

Tyson, NdeG., Strauss, M.A., Gott, J.R. (2016). Welcome To The Uniuverse. Princeton University Press. Woodstock, Oxfordshire, England. ISBN 9780691157245.

Vivid Maps. (2022). Martian Canals: A Short History of Martian Canals in Maps. Retrieved from: https://vividmaps.com/martian-canals/

Wikipedia. (2022). Tectonics of Mars. Retrieved from: https://en.wikipedia.org/wiki/Tectonics_of_Mars

Williams, D.R. (2022) Planetary Fact Sheet-NASA. Retrieved from: https://nssdc.gsfc.nasa.gov/planetary/factsheet/

Wolchover, N. (2012). Why is Mars Red? Retrieved from https://www.space.com/16999-mars-red-planet.html

Zhong, C. (2020). Formation and Change of Planetary Magnetic Field. American Journal of Astronomy and Astrophysics, 8(3), 61-65. https://doi.org/10.11648/j.ajaa.20200803.13

\section{Copyrights}

Copyright for this article is retained by the author(s), with first publication rights granted to the journal.

This is an open-access article distributed under the terms and conditions of the Creative Commons Attribution license (http://creativecommons.org/licenses/by/4.0/). 